Keywords: bowel cancer; bowel cancer screening; colorectal cancer screening; guaiac faecal occult blood test (gFOBt); nonuptake; socio-economic circumstances; qualitative

\title{
Reasons for non-uptake and subsequent participation in the NHS Bowel Cancer Screening Programme: a qualitative study
}

\author{
C K Palmer ${ }^{* 1}$, M C Thomas ${ }^{* 1}$, C von Wagner ${ }^{2}$ and R Raine ${ }^{1}$
}

${ }^{1}$ Department of Applied Health Research, University College London, 1-19 Torrington Place (Room 112), London WC1E 7HB, UK and ${ }^{2}$ Department of Epidemiology and Public Health, Cancer Research UK Health Behaviour Research Centre, University College London, 1-19 Torrington Place, London WC1E 6BT, UK

Background: Screening for bowel cancer using the guaiac faecal occult blood test offered by the NHS Bowel Cancer Screening Programme (BCSP) is taken up by $54 \%$ of the eligible population. Uptake ranges from $35 \%$ in the most to $61 \%$ in the least deprived areas. This study explores reasons for non-uptake of bowel cancer screening, and examines reasons for subsequent uptake among participants who had initially not taken part in screening.

Methods: Focus groups with a socio-economically diverse sample of participants were used to explore participants' experience of invitation to and non-uptake of bowel cancer screening.

Results: Participants described sampling faeces and storing faecal samples as broaching a cultural taboo, and causing shame. Completion of the test kit within the home rather than a formal health setting was considered unsettling and reduced perceived importance. Not knowing screening results was reported to be preferable to the implications of a positive screening result. Feeling well was associated with low perceived relevance of screening. Talking about bowel cancer screening with family and peers emerged as the key to subsequent participation in screening.

Conclusions: Initiatives to normalise discussion about bowel cancer screening, to link the BCSP to general practice, and to simplify the test itself may lead to increased uptake across all social groups.

Regular screening using the guaiac faecal occult blood test (gFOBt) reduces the risk of dying from bowel cancer by $16 \%$ (Hardcastle et al, 1996; Hewitson et al, 2008). Established in 2006, the English NHS Bowel Cancer Screening Programme (BCSP) initially offered biennial screening by gFOBt to all adults aged 60-69. Since 2010, screening began to be extended to those aged up to 74 . An analysis of uptake of the first 2.6 million screening invitations found overall uptake levels of $54 \%$ (von Wagner et al, 2011). This compares with overall uptake rates of $73 \%$ in the longer established NHS Breast Cancer Screening Programme (Health and Social Care Information Centre, 2013) and 79\% in the NHS Cervical Cancer Screening Programme (Health and Social Care Information Centre, 2012). Furthermore, a socio-economic gradient in gFOBt uptake exists which ranges from $35 \%$ in the most deprived areas to $61 \%$ in the least deprived areas (von Wagner et al, 2011).

Differences in uptake between socio-economic groups exist in other areas of health care and screening programmes (Goddard and Smith, 2001) but bowel cancer screening using gFOBt differs from the other NHS Cancer Screening Programmes in that it is self-completed. Eligible people receive a cardboard test kit through the post which is completed with smears of faeces taken from three separate bowel movements. The gFOBt is returned to the laboratory by post and participants receive the result within 2 weeks (Halloran, 2009). Studies primarily conducted in the United States report that reasons for not completing the gFOBt include feeling healthy, having no bowel symptoms, fear of the results of

*Correspondence: Dr CK Palmer; E-mail: cecily.palmer@ucl.ac.uk or MC Thomas; E-mail: mary.thomas@ucl.ac.uk

Received 23 October 2013; revised 7 February 2014; accepted 17 February 2014;

published online 11 March 2014

(c) 2014 Cancer Research UK. All rights reserved 0007-0920/14

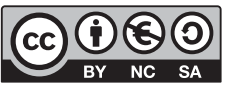

BJCN 0 
the screening test, and 'not wanting to know' (the latter often linked to doubts about the value of screening for the detection of health problems) (Beeker et al, 2000; McCaffery et al, 2001; Weitzman et al, 2001; Clavarino et al, 2004; Wackerbarth et al, 2005; Aubin-Auger et al, 2011). Difficulties in understanding the kit instructions, concerns about hygiene and storage of the kit, avoiding or delaying decision making, intention to take part but failure to do so for practical reasons, and a preference for a doctor to do such tests have also been identified as reasons for not completing screening (Dent et al, 1983; Szczepura et al, 2003; O'Sullivan and Orbell, 2004; Chapple et al, 2008; Hall et al, 2013). Of the four studies undertaken in the United Kingdom, two were conducted with individuals before being invited to be screened. Thus, they reported participants' beliefs about how they might behave rather than explanations based on actual experience (Szczepura et al, 2003; O'Sullivan and Orbell, 2004). The other two UK studies reported the views of participants who had been invited to participate in the NHS BCSP; in one, the majority of these participants had completed the gFOBt kit (Chapple et al, 2008), and the other reports on a relatively small sample restricted to one regional area (Hall et al, 2013).

We therefore have little understanding of the reasons for low uptake overall and for the social gradient in uptake of gFOBt in the English NHS BCSP. Furthermore, the NHS BCSP re-invites eligible adults every 2 years irrespective of whether or not they have previously responded and previous research has identified that repeated invitations increase uptake (Steele et al, 2010). Exploring reasons for subsequent participation among those who did not initially take up the screening invitation provides the unique opportunity to identify the tipping point to participation in screening and could inform interventions to address perceived barriers at the time of the first invitation. We therefore explored reasons for non-uptake of gFOBt among participants from diverse socio-economic backgrounds who had not accepted at least one screening invitation, and explored reasons for subsequent participation among participants who had not completed the gFOBt at an earlier invitation.

\section{MATERIALS AND METHODS}

Recruitment and sampling. The BCSP is delivered through five regional hubs in England. To recruit a socio-economically diverse range of participants, we used Index of Material Deprivation (IMD2010) scores to identify areas of South Yorkshire and London that embodied a spread of IMD scores, from the most to the least deprived. This index is a widely used area-based measure that combines seven domains (covering income deprivation, employment deprivation, health deprivation and disability, education skills and training deprivation, barriers to housing and services, living environment deprivation, and crime), into a single deprivation score for a small area (Department for Communities and Local Government, 2011). In collaboration with the two BCSP hubs covering South Yorkshire and London, 5100 individuals residing in areas including the most and least deprived as defined by IMD2010 who were recorded as having not accepted the invitation to screen on at least one occasion were invited to take part in a focus group held locally. Focus groups are of particular value when exploring reasons why people choose not to do something, or what Barbour terms 'why not' questions (Barbour, 2010). Respondents were allocated to one of sixteen focus groups (eight in Yorkshire and eight in London). Previous research found that focus groups benefit from being organised around the homogeneity of the participants (Bloor et al, 2001). Therefore, previous/current occupation has been used as a pragmatic indicator of socio-economic circumstances to allocate participants into groups with others of similar occupational and therefore socio-economic backgrounds (Craig and Forbes, 2005). Due to the potentially sensitive nature of the research topic, we also established separate focus groups for men and women.

In addition to participants who were recruited directly through the BCSP, and to more fully reflect the socio-demographic characteristics of the eligible screening population we also opportunistically recruited people of African-Caribbean origin through community settings in London. The use of an opportunistic approach was informed by previous studies which found that to explore Black and Minority Ethnic participants' experiences of health and health services may require alternative recruitment strategies to those used to recruit white European origin participants (McLean and Campbell, 2003; Twamley et al, 2010). An earlier study identified specific concerns about flexible sigmoidoscopy screening methodology held by men of AfricanCaribbean origin (McCaffery et al, 2001). We therefore sought to explore whether there were views and experiences of bowel cancer screening by gFOBt that were specific to this group. We held two focus groups, one with men and one with women of AfricanCaribbean ethnicity organised without reference to the occupational background of participants.

Data collection. Data were generated during 18 focus groups undertaken between July 2011 and April 2012. A topic guide was used to ensure that key topics were covered in each focus group. Groups were audio recorded and transcribed verbatim.

Analysis. Data were analysed inductively using techniques originating in grounded theory to generate themes from the data (Glaser and Strauss, 1967). Grounded theory describes an inductive approach to analysis in which data are used to derive and generate theory, in contrast to a deductive approach in which data are generated to test out a pre-existing theory (Murphy et al, 1998). By repeated close reading, and constant comparison of data, two researchers descriptively coded data as it was generated. Coded data were categorised into areas of thematic relevance to the research objectives. Through this iterative process and accompanied by discussion and writing, key themes were developed and refined, and links drawn between them. Comparative analyses were undertaken to determine whether themes specific to participants with non-professional compared with professional backgrounds (i.e., as an indicator of socio-economic difference) could be identified.

Ethics. The study received ethical approval from South East London research ethics committee five (reference 11/H0805/7), and NHS trust research governance was obtained at the relevant sites. Written informed consent was obtained from all participants.

\section{RESULTS}

Participant characteristics. In all, 243 individuals returned a completed consent form indicating willingness to take part in the research, of whom 128 subsequently took part in a focus group. The research team paid attention to ensure the sample included a balance of men $(n=67)$ and women $(n=61)$, and individuals with reported professional $(n=61)$ and non-professional $(n=50)$ occupational backgrounds (Table 1). Reported professional occupations included teacher, local government officer, solicitor, civil servant, nurse, dentist, journalist, artist, and social worker; and non-professional occupations included sales assistant, cook, cleaner, carer, builder, miner, driver, waitress, postman, and carpenter. In common with the purposively sampled focus groups, the opportunistically recruited focus groups comprising individuals of African-Caribbean origin included participants who had and had not taken part in bowel cancer screening. The majority of 


\begin{tabular}{|c|c|c|c|c|c|}
\hline & Location & $\begin{array}{c}\text { No. of } \\
\text { participants }\end{array}$ & $\begin{array}{l}\text { Occupational background } \\
\text { as indicator of } \\
\text { socio-economic circumstance }\end{array}$ & Sex & Ethnicity of participants \\
\hline FG01 & London & 11 & Professional & M & All White European \\
\hline FG02 & London & 5 & Non-professional & M & All White European \\
\hline FG03 & London & 8 & Professional & $\mathrm{F}$ & 7 White European, 1 South Asian \\
\hline FG04 & London & 6 & Non-professional & $\mathrm{F}$ & 5 White European, 1 African Caribbean \\
\hline FG05 & South Yorkshire & 7 & Professional & M & All White European \\
\hline FG06 & South Yorkshire & 8 & Non-professional & M & All White European \\
\hline FG07 & South Yorkshire & 7 & Professional & $\mathrm{F}$ & All White European \\
\hline FG08 & South Yorkshire & 6 & Non-professional & $\mathrm{F}$ & All White European \\
\hline FG09 & South Yorkshire & 7 & Non-professional & M & All White European \\
\hline FG10 & South Yorkshire & 8 & Professional & $\mathrm{M}$ & All White European \\
\hline FG11 & South Yorkshire & 6 & Non-professional & $\mathrm{F}$ & All White European \\
\hline FG12 & South Yorkshire & 6 & Professional & $\mathrm{F}$ & All White European \\
\hline FG13 & London & 6 & Non-professional & M & All White European \\
\hline FG14 & London & 8 & Professional & $\mathrm{M}$ & 7 White European, 1 West African \\
\hline FG15 & London & 6 & Non-professional & $\mathrm{F}$ & 4 White European, 2 African Caribbean \\
\hline FG16 & London & 6 & Professional & $\mathrm{F}$ & All White European \\
\hline FG17 & London & 10 & Not recorded & $\mathrm{F}$ & 9 African Caribbean, 1 South Asian \\
\hline FG18 & London & 7 & Not recorded & $\mathrm{M}$ & 6 African Caribbean, 1 West African \\
\hline
\end{tabular}

participants recalled receiving invitation(s) and gFOBt kit(s) from the BCSP. One hundred participants reported gFOBt non-uptake on at least one occasion, of whom 31 went on to complete the gFOBt kit when they were invited to take part in a subsequent screening round. Nine participants had not completed the gFOBt due to 'alternative uptake' of bowel cancer screening, such as colonoscopy, endoscopy, or gFOBt kit completion in primary or private care.

Our comparative analyses found high levels of consistency in accounts for non-uptake regardless of gender, ethnicity, or geographical location.

Themes. We present our findings as a series of six themes described below.

Themes common across non-professional and professional occupational groups:

Risks posed by faeces. Participants in all focus groups explained their aversion to complete a gFOBt kit by reference to the perceived risks that collecting, storing, and posting samples of faeces posed to hygiene. These risks were heightened by the requirement to complete the kit with samples from three separate bowel movements, which meant that the kit had to be stored over several days. Participants reported that the completion of the gFOBt kit threatened to physically pollute them or their environment, and that they would need to go to extreme lengths to manage these perceived threats.

'People's hands have to handle this yes? You don't know how strong germs get...so I don't fancy it going through the post.' (FG17P1)

'It's like sort of not flushing, only worse, it's sort of not nice...I wanted to scrub the bathroom down every day, so I thought it's not worth the hassle.' (FG03P5)

Completion of the gFOBt was considered to pose serious and fundamental threats to notions of socially acceptable and proper behaviour. Participants reported discomfort at the idea of handling faeces because this was an activity that was abnormal, broached a cultural taboo or could cause embarrassment and shame.

'You wouldn't normally leave faeces in your bathroom for three days.' (FG07P3)

'It's just not the done thing is it?'

'No - to mess about with it.' (FG08P5 + 6)

'What will happen in the bathroom with it... you know the whole complication of where to put it and if someone else walks in and finds it.' (FG16P4)

The perceived taboo of interacting with faeces was further illustrated by participants' concerns about being 'found out' to have completed the gFOBt. Being found to have stored or posted faecal samples was believed to be potentially socially and personally damaging, in that it could reflect badly on the individual and undermine them in the eyes of others. Some participants described the requirements of the gFOBt as 'offensive' and 'degrading'. The use of the term 'degradation' is of particular significance, because it carries ideas of personal cost in that it compromises the individual. Thus, completing the kit raised the threat of being at best embarrassed and at worst, disgraced and discredited.

'Put your poo in the post ... I thought oh god, y'know it's got your name on it and what if they open it.' (FG15P5)

'I've had two I've sent them both in the dustbin. I won't discuss it but I were a bit offended.' (FG09P6)

'I've had one and I ain't done it, I just felt degraded to tell you the truth.' (FG11P5)

The aversion to dealing with faeces that emerged in participants' accounts of non-uptake is underpinned by deeply ingrained definitions of faeces as a taboo substance (Thompson, 2013), 
and rigid social rules surrounding how it is appropriately dealt with.

Detachment from familiar health-care settings. Participants reported discomfort with the detachment of gFOB testing from 'usual' health-care settings and professionals. They expressed a preference to attend a health setting such as a GP surgery or hospital and for 'someone else' to undertake the screening on their behalf.

'Why don't they send you t'doctors or hospital to have it done there?...if the doctor sent for me and said I want to do so and so for you I'd go, or the hospital but doing that meself, I didn't like it at all.' (FG09P6)

On one level, participants linked their desire to 'go somewhere' such as a GP surgery or hospital with the avoidance of having to collect and sample their faeces. However, participants' references to medical settings as 'preferred' also revealed that the invitation to the BCSP was out of context and unsettling, because it required them to undertake a health procedure outside the settings in which health care is usually practiced. Further, linked to this was a perception of 'self-testing' as unusual and unexpected, particularly by comparison with other screening experiences or medical interactions where 'a professional' is involved in the procedure.

'I thought 'oh my god now we are asked to be doctors.' (FG16P6)

'I threw mine away, I'd rather have it done for me.'

'I'd rather go somewhere and have it done to be quite honest.' $(\mathrm{FG} 04 \mathrm{P} 5+6)$

Participants emphasised that it was unusual to have an active role in a health procedure, when the norm in medical encounters was for them to be the passive 'receiver' of care. They also disliked the impersonal nature of home testing:

'I would prefer my doctor to have some obvious interaction with me in the actual process rather than it being done with an anonymous third party.' (FG01P8)

By extension, it was noteworthy how many participants claimed that, had they been given an appointment to attend, or been told by their GP to complete a gFOBt kit, they would have done so. It appeared therefore that the detachment from clinical settings and professional roles may have reduced the perceived importance of the offer of screening.

'The message that was communicated to me was that this was hardly urgent or serious because if it was they would send me off to have a clinician do it.' (FG01P1)

'If the letter had come from my GP...I would have taken it more seriously.' (FG18P6)

The prospect of self-testing at home therefore inhibited rather than facilitated uptake.

The implications of knowing the screening results. The most complex theme to emerge related to the implications of knowing the screening results. Participants preferred not to be in possession of this information for several reasons. First, they commonly referred to the undesirable implications of a positive result. Thus, they expressed unwillingness to undergo the recommended procedures that may follow a positive gFOBt result, such as colonoscopy or bowel surgery. These participants often referred to previous experiences (their own or family members') of bowel investigations or treatment for gastroenterological problems, and described their negative consequences.

'It's the after effects, if they do find something, that would put me off taking the test in the first place... it's the colonoscopy, the treatment of the colonoscopy.' (FG10P3)

Non-uptake was therefore a means to protect oneself from the possible unpleasant consequences of a positive test.

Second, participants distinguished between 'being unwell' and 'knowing about being unwell'. A positive screening result meant that they would need to 'redefine' themselves as being unwell, which they did not wish to do because they believed it was unnecessary.

'If there's something the matter with me now, and I don't know about it, I'm fine. If somebody says I've got a problem, I'm going to worry about it, and I don't want that, you know you live life as it is now and I don't want people finding things.' (FG10P6)

Thus, there emerged from participants' accounts an alternative reading of screening as an activity that, rather than maintaining good health, may actually be complicit in generating ill health. By presenting screening as a process that could undermine health, and questioning the value of the knowledge offered by screening to the maintenance of good health, participants pointed out that the benefit of declining to complete the gFOBt allowed one to 'get on with life'.

'This is just like sticking your head down the loo and thinking you've got cancer all day, you know there's a balance of how much 'into' things you should get.' (FG03P3)

'To me it's like mollycoddling yourself so everything working right, don't mess about with yourself.' (FG05P1)

Finally, the possibility that screening might identify cancer and result in subsequent interventions was described by some participants as too frightening to contemplate. The knowledge offered by screening was for some a stressful and frightening prospect, to the extent that actively choosing not to be in possession of this information was preferable.

'I'm scared, simple as that...it's the test coming back positive that worries me, so I tend to ignore it and hope it goes away.' (FG02P2)

\section{Researcher: 'What's frightening?'}

Participant: 'What actually might be discovered, what I don't know is not there like, if you know what I mean.' (FG13P3)

Thus, some participants demonstrated an ambivalence towards, or overt rejection of the knowledge offered by screening. Analysis of the accounts of participants who 'didn't want to know' found that it was also common for such participants to describe cancer as a particularly serious and frightening diagnosis for which treatment was unpleasant and often futile. Participants' attitudes towards cancer treatment further underpinned their rejection of the knowledge offered by screening, because, if there was perceived to be little benefit associated with treatment, there was little point in taking part in screening.

'[A friend] went through all that chemotherapy and all that suffering it didn't make a... difference.' (FG08P6) 
Judgements of good health and low relevance of screening. Many participants believed that the gFOBt was irrelevant because they were certain that they did not have and were unlikely to get bowel cancer. The evidence they cited included a lack of symptoms, being physically active and having no family history of bowel cancer.

'I've got no symptoms so I'm alright, y'know, I go to the toilet regular and y'know, I exercise and I'm fit.' (FG09P1)

Descriptions of being in good health were often interwoven with other themes of non-uptake.

Themes present among professional occupational groups only: Delaying uptake, leading to non-uptake. No themes emerged solely from participants with non-professional backgrounds. However, we identified one theme associated with non-uptake which was discussed only by participants with professional backgrounds. These respondents commonly described their nonuptake in terms of delay, rather than outright rejection. Participants reported that the gFOBt was 'put to one side', or 'put in the in-tray' implying some degree of intention to participate, but ultimately kits were not completed. Delay was often linked to descriptions of the complexity of the instructions for completing the gFOBt, and also the time-consuming nature of kit completion.

'You've got to really sit down and read it, y'can't, it's not just something you can pick up and say 'oh I'll go and do that now', you've got to study it.' (FG12P4)

'It's quite a long winded, drawn out thing I just kept putting off doing it.' (FG03P8)

There was a common misconception among participants in all focus groups that samples had to be taken on 3 consecutive days. Respondents from professional backgrounds cited this rigid, 3-day 'window' for test completion as a cause of delay and subsequent non-completion because it was not possible to fit the test requirements in with their bowel movements or routine and lifestyle.

'If I start it on one day I've got to remember then to do it for the next two days and that was a big block for me because I'm very rarely in the same place for three days in a row.' (FG14P8)

Non-uptake followed by uptake in a subsequent screening round:

The power of talk: a key 'tipping point'. Participants from all occupational backgrounds who reported that they had not initially participated in screening and had then completed the gFOBt in a subsequent screening round described being influenced by discussions with family members, friends, and health professionals. They reported being questioned about their initial refusal to complete the test or being told outright to take part in bowel cancer screening. They also recalled supportive discussions in which their concerns about or aversions to the gFOBt kit were discussed and challenged. Participants reported that becoming aware that their partner or friends had already completed the gFOBt was influential. In addition, they reported that becoming aware that a family member or friend had developed bowel cancer influenced them to take part in screening.

'My brother in law was diagnosed with bowel cancer after I'd had the first request which I totally ignored... my wife did [her gFOBt] and she got her results back which were clear, peace of mind, I thought well you silly bugger, you know why didn't I do [it]?' (FG09P4)
'A friend also had it and she was telling me about how she did it and I thought gosh it's not as complex as I think.' (FG16P2)

Discussions in which other individuals championed participation in screening, or revealed their own gFOBt uptake was repeatedly implicated by participants as the key tipping point to a decision to undertake screening on a subsequent occasion. Through talk with others, participants described themselves as 'nagged', encouraged and reassured to undertake the gFOBt. Furthermore, through talking with others, and becoming aware of others' completion of the gFOBt, uptake was repositioned as a normal activity. The particular power of talk appeared to normalise the unusual, unexpected and potentially taboo aspects of the gFOBt kit.

'I think as well it's a critical mass isn't it, so you discover your friends are all doing it or whatever so then it does become a slightly normal thing to do.' (FG16P1)

\section{DISCUSSION}

Summary of principal findings. The multifaceted explanations for non-uptake of gFOBt that we found do not, in the main, vary according to individuals' socioeconomic backgrounds. In addition to practical objections (a perceived hygiene risk, complex or unfeasible instructions and process), we demonstrate that gFOBt completion posed threats to social self (by breaking social taboos surrounding faeces that might bring embarrassment and shame on the participant) (Lawton, 1998; Thompson, 2013). Furthermore, it posed threats to participants' wellbeing (e.g., by redefining well people as ill, and leading to unwanted investigations). Self-testing at home and beliefs about low personal risk of developing bowel cancer undermined the value and relevance of screening. However, talk increased awareness of screening uptake by peers and significant others and was key to overcoming objections, and to subsequent screening participation.

Comparison with other research. Our finding that non-uptake is linked to detachment of gFOBt from clinical settings is a novel finding, which has not to our knowledge been reported previously in the United Kingdom. An Australian study from the 1980s reported that some people declined doing a gFOBt because they wanted their own doctors to do such tests (Dent et al, 1983). This may explain why general practitioner endorsement of bowel cancer screening invitation letters can increase screening uptake (Cole et al, 2002; Hewitson et al, 2011). Previous studies report that participants downplay concerns relating to handling faecal matter, or frame such concerns solely in terms of hygiene risks (Weitzman et al, 2001; O'Sullivan and Orbell, 2004; Chapple et al, 2008). We demonstrate the greater complexity of this concern in terms of its relationship to shame, embarrassment, and the broach of cultural taboos. Our more extensive findings are likely to be explained by our recruitment criteria that allowed us to explore reasons for nonparticipation in screening among participants who had been invited to the NHS BCSP on more than one occasion. In contrast, although Chapple et al (2008) interviewed people who either delayed or who had refused screening they also included many people who took part in screening without delay. Other previous studies included people who had not yet received a screening invitation from the BCSP (Weitzman et al, 2001; O'Sullivan and Orbell, 2004).

In common with previous research, we identified non-uptake as preferable to being in possession of the information offered by screening due to the potential negative implications of a positive gFOBt result (McCaffery et al, 2001; Weitzman et al, 2001; O'Sullivan and Orbell, 2004; Chapple et al, 2008). Previous studies 
have also documented the belief that screening is unnecessary for asymptomatic, fit individuals with no family history (Beeker et al, 2000; McCaffery et al, 2001; Clavarino et al, 2004; Chapple et al, 2008). The inconvenience of gFOBt completion and mistaken belief that samples had to be collected on 3 consecutive days has also been reported previously (Beeker et al, 2000; Chapple et al, 2008). Finally, previous research highlights the difficulties of talking openly about bowel cancer (Beeker et al, 2000; Szczepura et al, 2003; O'Sullivan and Orbell, 2004). However, our finding about the impact of talk with others on subsequent screening uptake resonates with the proposal in Beeker et al (2000), that greater cultural visibility of colorectal cancer screening, for example in the mass media, may provide people with the "permission,' motivation and the tools' to discuss this culturally taboo topic and to normalise test completion as an everyday activity.

These qualitative data illustrate how core beliefs described in health behaviour models (e.g., the Health Belief Model; Rosenstock, 1974) manifest themselves in the context of bowel screening. First, issues around faecal sampling represented an important emotional barrier to bowel screening. Second, the importance of perceived susceptibility in cancer screening was illustrated by the theme that screening is not relevant among those who regard themselves as healthy. Finally, the power of talk highlights the importance of providing external (social) cues to action in addition to routine invitations and reminders. Specifying core beliefs is instrumental in developing theory-based interventions to increase uptake.

Strengths and limitations. This is the largest in-depth exploration of non-uptake of bowel cancer screening and the first study to explore why non-participants subsequently undergo screening. Knowledge about the determinants of behaviour changes provides foci for the content of strategies to increase uptake. However, we were unable to delineate specific reasons for lower uptake among more socially disadvantaged groups. This may be in part because of the existence of a social gradient in uptake rather than a gap between two discrete social groups for whom there may be distinct explanations for their behaviour. It may also have been in part due to our inability to explore a potential link between literacy levels and uptake. Finally, because our sample did not include people with chronic illnesses we could not explore the role of poor health (and associated functional impairments) in uptake.

Implications for UK policy and practice. Our results suggest that strategies to increase gFOBt uptake in all social groups should include three components. First, initiatives to normalise open discussions about bowel cancer screening should be designed and evaluated, such as the advertising campaign on-going in Greater Manchester to encourage people to talk about the BCSP (Greater Manchester Bowel Cancer Screening Health Improvement Team, 2012). Second, there should be explicit linkage of the BCSP to general practice. Endorsement of invitation, letters either by the medical practice or by an individual general practitioner is known to be effective (Cole et al, 2002; Hewitson et al, 2011). We are currently experimentally evaluating an amendment to the BCSP invitation letter which simply states that the individual's named general practice endorses the invitation, and some areas are using financial incentives to encourage GPs to follow-up patients who have not accepted a screening invitation. Third, existing information materials require amendment to clarify the advantages of early diagnosis in asymptomatic individuals. In addition, the singlesample faecal immunochemical test (FIT) is due to be piloted within the BCSP in 2014. If effective, then it should address obstacles associated with the complexity and storage of the current gFOBt. The relationship between literacy and uptake requires further exploration through the experimental comparison of new information designed to address readability and comprehension in comparison with existing BCSP materials.
It is noteworthy that while our study identifies reasons for nonuptake specific to bowel cancer screening, it also reports reasons for non-uptake (implications of knowing screening results; judgements of good health; low relevance of screening), which could be considered more generic and relevant to other screening programmes.

\section{CONCLUSIONS}

Explanations for non-uptake of the gFOBt do not, in the main, vary according to individuals' socioeconomic backgrounds. Strategies to improve uptake of bowel cancer screening should incorporate initiatives to normalise open discussion about bowel cancer screening; explicitly link the BCSP to general practice; modify existing BCSP materials to state the advantages (and risks) of early diagnosis in asymptomatic individuals; and simplify the screening test itself.

\section{ACKNOWLEDGEMENTS}

We are grateful to Directors and staff of the Bowel Cancer Screening Programme hubs, and individuals representing community-based groups for their help with the recruitment of participants. We further thank all those who gave up their time to take part in a focus group.

This article presents independent research funded by the National Institute for Health Research (NIHR) under its Programme Grants for Applied Research Programme (RP-PG0609-10106). The views expressed in this article are those of the authors and not necessarily those of the NHS, the NIHR or the Department of Health. NIHR had no role in study design, data collection, analysis or interpretation, writing of the paper or the decision to submit this paper for publication. The study sponsor is University College London.

\section{CONFLICT OF INTEREST}

The authors declare no conflict of interest.

\section{REFERENCES}

Aubin-Auger I, Mercier A, Lebeau JP, Baumann L, Peremans L, Van Royen P (2011) Obstacles to colorectal screening in general practice: a qualitative study of GPs and patients. Fam Pract 28(6): 670-676.

Barbour R (2010) Focus groups. In: Bourgeault I, Dingwall R, de Vries R (eds). The Sage Handbook of Qualitative Methods in Health Research. Sage Publications: London, 2010, pp 327-352.

Beeker C, Kraft JM, Southwell BG, Jorgensen CM (2000) Colorectal cancer screening in older men and women: qualitative research findings and implications for intervention. J Commun Health 25(3): 263-278.

Bloor M, Frankland J, Thomas M, Robson K (2001) Focus Groups in Social Research. Sage: London.

Chapple A, Ziebland S, Hewitson P, McPherson A (2008) What affects the uptake of screening for bowel cancer using a faecal occult blood test (FOBt): a qualitative study. Soc Sci Med 66(12): 2425-2435.

Clavarino AM, Janda M, Hughes KL, Del Mar C, Tong S, Stanton WR, Aitken JF, Leggett BA, Newman B (2004) The view from two sides: a qualitative study of community and medical perspectives on screening for colorectal cancer using FOBT. Prev Med 39(3): 482-490.

Cole SR, Young GP, Byrne D, Guy JR, Morcom J (2002) Participation in screening for colorectal cancer based on a faecal occult blood test is improved by endorsement by the primary care practitioner. J Med Screen 9(4): 147-152.

Craig P, Forbes J (2005) Social position and health: are old and new occupational classifications interchangeable? J Biosoc Sci 37(1): 89-106. 
Dent OF, Bartrop R, Goulston KJ, Chapuis PH (1983) Participation in faecal occult blood screening for colorectal cancer. Soc Sci Med 17(1): 17-23.

Department for Communities and Local Government (2011) The English Indices of Deprivation 2010. https://www.gov.uk/government/uploads/ system/uploads/attachment_data/file/6320/1870718.pdf (accessed September 2013).

Glaser BG, Strauss AL (1967) The Discovery of Grounded Theory: Strategies for Qualitative Research. Aldine: New York.

Goddard M, Smith P (2001) Equity of access to health care services: theory and evidence from the UK. Soc Sci Med 53(9): 1149-1162.

Greater Manchester Bowel Cancer Screening Health Improvement Team (2012) 'Talk about it' media campaign. http://www.bowelscreening.org/ index.php/latest-news/95-talking-saves-lives.html.

Hall NJ, Rubin GP, Dobson C, Weller D, Wardle J, Ritchie M, Rees CJ (2013) Attitudes and beliefs of non-participants in a population-based screening programme for colorectal cancer. Health Expect; e-pub ahead of print 25 November 2013; doi:10.1111/hex.12157.

Halloran S (2009) Bowel cancer screening. Surgery 27: 397-400.

Hardcastle JD, Chamberlain JO, Robinson MH, Moss SM, Amar SS, Balfour TW, James PD, Mangham CM (1996) Randomised controlled trial of faecal-occult-blood screening for colorectal cancer. Lancet 348(9040): 1472-1477.

Health and Social Care Information Centre (2013) Breast Screening Programme - England, 2011-2012. https://catalogue.ic.nhs.uk/ publications/screening/breast/bres-scre-prog-eng-2011-12/bres-screprog-eng-2011-12-rep.pdf (accessed September 2013).

Health and Social Care Information Centre (2012) Cervical Screening Programme - England, 2011-2012. https://catalogue.ic.nhs.uk/ publications/screening/cervical/cerv-scre-prog-eng-2011-12/ cerv_scre_prog_eng_2011-12_rep_v3.pdf (accessed September 2013).

Hewitson P, Ward AM, Heneghan C, Halloran SP, Mant D (2011) Primary care endorsement letter and a patient leaflet to improve participation in colorectal cancer screening: results of a factorial randomised trial. Br J Cancer 105(4): 475-480.

Hewitson P, Glasziou P, Watson E, Towler B, Irwig L (2008) Cochrane systematic review of colorectal cancer screening using the fecal occult blood test (hemoccult): an update. Am J Gastroenterol 103(6): $1541-1549$.

Lawton J (1998) Contemporary hospice care: the sequestration of the unbounded body and 'dirty dying'. Sociol Health Illn 20(2): 121-143.

McCaffery K, Borril J, Williamson S, Taylor T, Sutton S, Atkin W, Wardle J (2001) Declining the offer of flexible sigmoidoscopy screening for bowel cancer: a qualitative investigation of the decision-making process. Soc Sci Med 53(5): 679-691.
McLean C, Campbell C (2003) Locating research informants in a multi-ethnic community: ethnic identities, social networks and recruitment methods. Ethn Health 8(1): 41-61.

Murphy E, Dingwall R, Greatbatch D, Parker S, Watson P (1998) Qualitative research methods in health technology assessment: a review of the literature. Health Technol Assess 2: 16.

O'Sullivan I, Orbell S (2004) Self-sampling in screening to reduce mortality from colorectal cancer: a qualitative exploration of the decision to complete a faecal occult blood test (FOBT). J Med Screen 11(1): $16-22$.

Rosenstock IM (1974) Historical origins of the health belief model. Health Educ Monogr 2: 1-8.

Steele R, Kostourou I, McClements P, Watling C, Libby G, Weller D, Brewster DH, Black R, Carey FA, Fraser C (2010) Effect of repeated invitations on uptake of colorectal cancer screening using faecal occult blood testing: analysis of prevalence and incidence screening. BMJ 341: c5531.

Szczepura A, Johnson M, Orbell S, Gumber A, O’Sullivan I, Clay D, Owen D (2003) Ethnicity: UK Colorectal Cancer Screening Pilot: Final Report. http://www.cancerscreening.nhs.uk/bowel/ethnicity-finalreport.pdf (accessed September 2013).

Thompson AI (2013) 'Sometimes, I think I might say too much': dark secrets and the performance of inflammatory bowel disease. Symb Interact 36(1): 21-39.

Twamley K, Puthussery S, Macfarlane AJ, Harding S, Baron M (2010) Recruiting UK-born women from ethnic minority groups for qualitative health research - lessons learned from a study on maternity care. Res Policy Planning 15(3): 156-162.

von Wagner C, Baio G, Raine R, Snowball J, Morris S, Atkin W, Obichere A, Handley G, Logan RF, Rainbow S, Smith S, Halloran S, Wardle J (2011) Inequalities in participation in an organized national colorectal cancer screening programme: results from the first 2.6 million invitations in England. Int J Epidemiol 40(3): 712-718.

Wackerbarth SB, Peters JC, Haist SA (2005) 'Do we really need all that equipment?': factors influencing colorectal cancer screening decisions. Qual Health Res 15(4): 539-554.

Weitzman ER, Zapka J, Estabrook B, Goins KV (2001) Risk and reluctance: understanding impediments to colorectal cancer screening. Prev Med 32(6): 502-513.

(c) (1) (2) This work is licensed under the Creative Commons BY NC SA Attribution-NonCommercial-Share Alike 3.0 Unported License. To view a copy of this license, visit http://creativecommons. org/licenses/by-nc-sa/3.0/ 